\title{
A high frequency search for highly dispersed pulsars
}

\author{
M. Kramer, A. Jessner, P. Müller and R. Wielebinski \\ Max-Planck-Institut für Radioastronomie, Bonn, Germany
}

\section{Introduction}

The majority of known pulsars have been discovered by pulsar searches at low radio frequencies $(\nu<1 \mathrm{GHz})$. However, such searches are subject to various deleterious effects, viz the Galactic background radiation $\left(\propto \nu^{-2.8}\right)$, dispersion smearing $\left(\propto \nu^{-3}\right)$ and also scatter broadening $\left(\propto \nu^{-4.4}\right)$. Dispersion smearing and, in particular, scatter broadening prohibit the detection of pulsars with high dispersion measures at low frequencies (cf. Fig. 1a). This is highlighted by the fact that all 11 known pulsars with $\mathrm{DM}>600 \mathrm{~cm}^{-3} \mathrm{pc}$ have been discovered during the only two surveys performed to date above $1 \mathrm{GHz}$, i.e. at $1.4 \mathrm{GHz}$ by Clifton et al. (1992) and at $1.5 \mathrm{GHz}$ by Johnston et al. (1992). However, scattering is still a limiting factor at even $1.4 / 1.5 \mathrm{GHz}$. For example B1750-24 is observed with a double component profile at $4.85 \mathrm{GHz}$ (Kijak et al. 1996), whereas at $1.4 \mathrm{GHz}$ the components are completely smeared out due to scatter broadening (cf. Clifton et al. 1992). Therefore, the galactic population of highly dispersed pulsars is still not known. In order to reveal this hidden sample, we have recently started a search in Effelsberg at $4.85 \mathrm{GHz}$ where limitations due to scattering are essentially not existent (see Fig. 1a). The use of this extraordinary high frequency for pulsar searches enables us to observe with a large bandwidth but a small number of filterbank channels, so that the necessary computer power is radically reduced. However, the general steepness of pulsar spectra demands a highly sensitive observing system, otherwise, only the most luminous sources can be detected. A serious disadvantage of a high frequency search is the small telescope beam requiring a lot of observing time to search even a small area of the sky. A restriction of the search area is therefore highly recommended.

\section{Observing system and data analysis}

We use a high sensitivity $4.85 \mathrm{GHz}$-HEMT-receiver recently installed in the secondary focus of the Effelsberg $100 \mathrm{~m}$-radiotelescope. The system temperature was found to be less than $30 \mathrm{~K}$ on the sky in zenith position. The gain is still large, i.e. $1.45 \mathrm{~K} / \mathrm{Jy}$, while the beamsize drops to $\sim 150^{\prime \prime}$. The total bandwidth of $480 \mathrm{MHz}$ of LHC and RHC polarized signals is divided into eight channels of $60 \mathrm{MHz}$ bandwidth. The outputs of all sixteen channels are detected and digitized by $2-\mathrm{MHz} \mathrm{V} / \mathrm{f}$-converters. A multiplexer following the filter bank pairs the channels of equal frequency digitally and compresses these eight total power outputs by a digital time-differentiation. Two adjacent channels are bit pattern encoded and supplied to the parallel inputs of the standard pulsar backend. Since we primarily expect to detect relatively young pulsars, i.e. $P \geq 20 \mathrm{~ms}$, the 

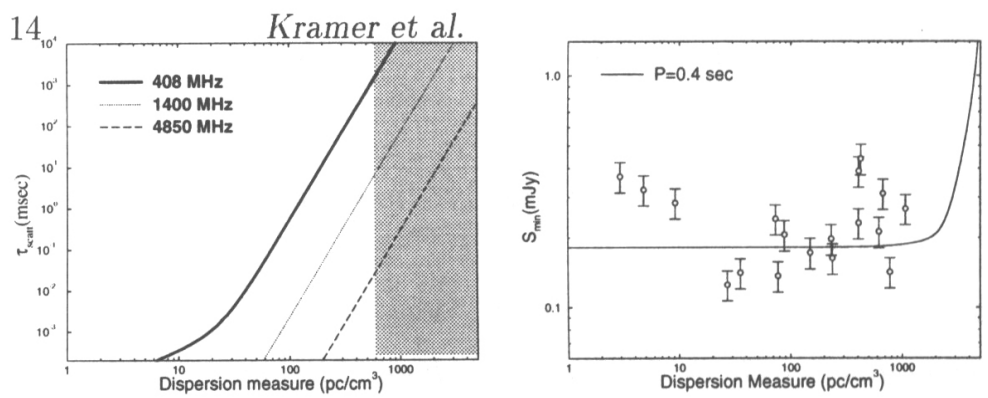

Figure 1. a) Scatter broadening as a function of DM for different frequencies (derived from Bhattacharya 1992 and Cordes et al. 1985), b) measured system sensitivity scaled to detection threshold of $\mathrm{S} / \mathrm{N} \sim 8$. The solid line is derived for the average period of our sample $P \sim 0.4$ s.

data are sampled every $500 \mu \mathrm{s}$ for typically $9 \mathrm{~min}$, i.e. $2^{20}$ samples. The data are written to disk before they are reduced off-line by an HP-Apollo 9000/735 (272 MByte RAM) using software based on a programme obtained by courtesy of J.H. Taylor and the Princeton Pulsar Group (cf. Nice 1992).

\section{Test observations and results}

In order to test the developed hard- and software, and to calibrate the system sensitivity, we observed known sources covering periods between $0.085 \mathrm{~s} \leq \mathrm{P} \leq$ $1.5 \mathrm{~s}$ and $\mathrm{DMs}$ between $0 \leq \mathrm{DM} \leq 1074 \mathrm{~cm}^{-3} \mathrm{pc}$. We first observed each source in a. standard observing mode (i.e. synchronously folding at the pulse period) in order to accurately measure the corresponding flux density, before we switched to search mode within a few seconds. We were able to detect all known high DM pulsars, showing the system's capability to look for yet unknown highly dispersed pulsars in the Galaxy. We easily detected, for instance, B1758-23, which has presently the largest known DM of $1074 \mathrm{~cm}^{-3} \mathrm{pc}$, with a $\mathrm{S} / \mathrm{N}$ of 14 . The sensitivity was calibrated to be less than $0.2 \mathrm{mJy}$ for an integration time of $9 \mathrm{~min}$, independent of dispersion measures below $3000 \mathrm{~cm}^{-3} \mathrm{pc}$ (Fig. 1b). The band was fortunately found to be free of strong interference. The system is now used for an ongoing search for pulsars along the plane of the inner Galaxy.

Acknowledgments. We would like to thank a number of people who have been involved in this project. We in particular mention F. Camilo, J.A. Gil, O. Lochner, A. Schmidt, U. Teuber, J.H. Taylor, H. Wiedenhöver, W. Wiedenhöver and W. Zinz. We are grateful to D. Lorimer for extremely helpful comments.

\section{References}

Bhattacharya D., et al. 1992, A\&A, 254, 198

Cordes J.M.:, Weisberg J.M., Boriakoff V. 1985, ApJ, 288, 221

Clifton T.R., et al. 1992, MNRAS, 254, 177

Johnston S., et al. 1992, MNRAS, 255, 401

Kijak J., et al. 1996, in prep.

Nice D.J., 1992, Ph.D. thesis, Princeton University 